\title{
A comparative study of Sargassum horneri Korea and China strains collected along the coast of Jeju Island South Korea: its components and bioactive properties
}

\author{
Hyun-Soo Kim ${ }^{1}$, K. K. Asanka Sanjeewa ${ }^{1}$, I. P. Shanura Fernando ${ }^{1}$, BoMi Ryu ${ }^{1}$, Hey-Won \\ Yang ${ }^{1}$, Ginnae Ahn' ${ }^{2}$, Min Cheol Kang ${ }^{1}$, Soo-Jin Heo ${ }^{3}$, Jun-Geon Je ${ }^{1}$ and You-Jin Jeon, ${ }^{1, *}$ \\ ${ }^{1}$ Department of Marine Life Science, School of Marine Biomedical Sciences, Jeju National University, Jeju 63243, Korea \\ ${ }^{2}$ Department of Marine Bio-Food Sciences, Chonnam National University, Yeosu 59626, Korea \\ ${ }^{3}$ Jeju International Marine Science Center for Research \& Education, Korea Institute of Ocean Science and Technology, Jeju \\ 63349, Korea
}

Sargassum horneri is edible brown seaweed abundant along the coasts of Jeju Island, South Korea. In addition to the native $S$. horneri population, a large amount of $S$. horneri has been found to invade Jeju Island from the east coast of China. Thus, S. horneri of both Korea (SK) and China (SC) strains now inhabits along with the shore of Jeju Island and have become a threat to the coastal biodiversity. However, they could be used in obtaining functional ingredients for industrial level applications provided an optimized cost effective strategy. In the present study, we compared SK and SC strains for the extraction efficiency, components, antioxidant, and anti-inflammatory properties of $80 \%$ methanolic extracts and their partially purified fractions. According to the results, two strains indicated similar bioactive properties such as DPPH and alkyl radical scavenging activity as well as anti-inflammatory activities on lipopolysaccharide-stimulated RAW 264.7 cells. The yield of $80 \%$ methanol extract from SC was higher than SK. However, the yields of the ethyl acetate and chloroform fractions from SK were higher than those of SC strain. The major peaks in the high-performance liquid chromatography chromatograms, which was identified as Apo-9 fucoxanthinone, indicated that both methanolic extracts of SK and SC contains major target peaks but with different amounts. This study might be useful for developing functional materials from SC and SK in future.

Key Words: anti-inflammatory; antioxidant; Sargassum horneri

\section{INTRODUUCTION}

Reactive oxygen species (ROS), such as superoxide radicals, hydroxyl radical, and hydrogen peroxide are generated inside the cells during the physiological processes. ROS are a group of oxygen containing compounds that are highly reactive towards essential biomolecules, which in turn interfere with normal cellular functionalities (Fer- nando et al. 2016). Cellular systems are equipped with enzyme-mediated antioxidant defense mechanisms to maintain ROS in safe levels. However, the imbalances between ROS production and antioxidant defense systems results in oxidative stress (Fernando et al. 2017a). Excessive and prolonged production of ROS are responsible for
(7) \$ This is an Open Access article distributed under the terms of the Creative Commons Attribution Non-Commercial License (http://creativecommons.org/licenses/by-nc/3.0/) which permits unrestricted non-commercial use, distribution, and reproduction in any medium, provided the original work is properly cited.
Received February 13, 2018, Accepted November 15, 2018

* Corresponding Author

E-mail: youjinj@jejunu.ac.kr

Tel: +82-64-754-3475, Fax: +82-64-756-3493 
the pathogenesis of disease conditions such as cancer, inflammation, cardiovascular diseases, and diabetes (Lin and Beal 2006).

Inflammation is a protective response that plays a key role in host defense against pathogens. However, uncontrolled inflammatory responses can cause the pathogenesis of disease conditions such as cancer, obesity, metabolic syndrome, and type 2 diabetes (Esser et al. 2014, Pesic and Greten 2016). Hence evaluation of extracts and purified compounds for antioxidant and antiinflammatory functionality receive a special attention in natural product research. Many of the natural products are biocompatible and safe compared to the synthetic drugs. Seaweeds have been identified as a bioresource rich in bioactive compounds with a wide diversity of functional properties (Choi et al. 2016, Park et al. 2016, Sanjeewa et al. 2017a). Seaweeds are rich in polyphenols, polysaccharides, terpenoids, and unsaturated fatty acids with bioactive properties (Sanjeewa et al. 2016, Fernando et al. 2017b). This enables them to be used in the development of functional ingredients in nutraceuticals, cosmeceuticals, and functional foods (Wijesinghe and Jeon 2012). Sargassum horneri is an edible brown seaweed inhabiting the coasts of Korea, Japan, and China (Pang et al. 2009, Sanjeewa et al. 2017a). It grows abundantly attached on rocks in the upper and middle intertidal zones. In contrast to the surface attached strains, some S. horneri strains have spherical gas-filled bladders, which gain them the ability to float on the sea surface (Wang et al. 2014, Sanjeewa et al. 2018). S. horneri is one of the ecologically-important seaweed types which provides crucial habitats for unique and specialized groups of marine animals and other plants in the ocean ecosystems (Wang et al. 2014). In contrast to the ecological importance, this seaweed is also considered as a rich source of nutrients such as dietary fibers, vitamins, amino acids, and polysaccharides. In addition to its nutraceutical value, it is utilized as a functional material in traditional medicine for thousands of years in Asian countries such as Korea, China, and Japan (Preeprame et al. 2001, Kubo et al. 2016, Sanjeewa et al. 2017b). Several studies conducted during the last few decades report $S$. horneri as a source rich in bioactive compounds that includes sulfated polysaccharides and phlorotannins (Preeprame et al. 2001, Yoshioka et al. 2014, Sanjeewa et al. 2017a). Jeju is located in the East China Sea $\sim 100 \mathrm{~km}$ from south of Korea Peninsula, $\sim 200 \mathrm{~km}$ west of Kyushu Island in Japan, $\sim 500 \mathrm{~km}$ east of Jiangsu Province or Shanghai in China, and $\sim 1,000 \mathrm{~km}$ northeast of Taiwan (Kundu and Kawamura 2014). In addition to the local population of S. horneri, a consid- erable amount of S. horneri China strain (SC) is moving to the coasts of Jeju Island from the east coast of China with ocean currents. Thus, large quantities of Korea and China strains are available along the Coast of Jeju Island (Sanjeewa et al. 2017a). However, these algae have not studied in much detail for their bio-functional natural products. The present study was undertaken to compare the antioxidant and anti-inflammatory properties of $80 \%$ methanol extracts of the two $S$. horneri strains and their solvent fractions.

\section{MATERIALS AND METHODS}

\section{Materials}

The murine macrophage cells RAW 264.7 was purchased from the Korea Cell Line Bank (Seoul, Korea). The Dulbecco's modified Eagle's medium (DMEM), fetal bovine serum (FBS) and penicillin-streptomycin, were purchased from Gibco BRL (Burlington, ON, Canada). Dimethyl sulfoxide (DMSO), 3-(4,5-Dimethylthiazol2-yl)-2,5-diphenyltetrazolium bromide (MTT), 2,2-diphenyl-1-picrylhydrazy (DPPH), and 2,2'-azobis (2-amidinopropane) hydrochloride (AAPH) were purchased from Sigma-Aldrich (St. Louis, MO, USA). All solvents used for the preparation of crude samples were of analytical grade (Dae-Jung Chemicals \& Metals, Seoul, Korea). High-performance liquid chromatography (HPLC) grade solvents were purchased from Burdick \& Jackson (Muskegon, MI, USA).

\section{Sample collection and preparation}

S. horneri samples were collected during the winter season in April 2015 along the shores of Jeju Island, Korea. The seaweed species were identified by Jeju Biodiversity Research Institute (Jeju, Korea). Two strains were identified by molecular level identification (data not given). The seaweed samples were washed with running tap water to remove any salt, epiphytes, and sand. The washed S. horneri samples were frozen at $-80^{\circ} \mathrm{C}$, lyophilized and ground to a powder.

\section{Sample preparation and fractionation}

The freeze dried seaweed powder (50 g) was extracted three times with $80 \%$ methanol (1 L) at room temperature, filtered under vacuum and concentrated using the rotary evaporator. The obtained crude was suspended in 
distilled water and partitioned between n-hexane, chloroform, and ethyl acetate, according to the polarity. The resulting solvent fractions were concentrated using the rotary evaporator. The extracts were dissolved in DMSO, for the cell culture experiments and diluted using phosphate buffered saline. The final concentration of DMSO in the culture medium was less than $0.1 \%$.

\section{Chemical analysis}

The ash content of freeze-dried seaweed samples was determined by dry ashing in a furnace at $550^{\circ} \mathrm{C}$ for $6 \mathrm{~h}$ (Association of Official Analytical Chemists 1998). Moisture content of seaweeds were determined by using the oven-drying method at $105^{\circ} \mathrm{C}$ in the moisture analyzer (mb45; OHAUS, Nänikon, Switzerland). Crude protein content of seaweeds were measured by Kjeldahl digestion method. Crude lipid content of seaweed samples were measured by Soxhlet method with diethyl ether as the solvent (Soxtec 2050; FOSS Analytical, Hillerød, Denmark) (Chandler and Dodds 1983). The total polysaccharide contents of $80 \%$ ethanolic extracts were analyzed using the phenol-sulfuric acid according to the method described by DuBois et al. (1956). The total polyphenol content of extracts was analyzed according to the method described by Chandler and Dodds (1983).

\section{HPLC analysis of seaweed extracts}

HPLC analysis was carried out using an Atlantis T3 ODS $3 \mu \mathrm{m}, 3.0 \times 150 \mathrm{~mm}$ column (Waters Corporation, Milford, MA, USA) connected to a photodiode array detector. The column was eluted in gradient mode with a mobile phase solvent system containing acetonitrile and water (acetonitrile with $0.1 \%$ formic acid-water with $0.1 \%$ formic acid [0-50 min: $5: 95-95: 5 \mathrm{v} / \mathrm{v}$; 50-60 min: 100 : $0 \mathrm{v} / \mathrm{v}]$ ) with a flow rate of $0.3 \mathrm{~mL} \mathrm{~min}^{-1}$, and the ultraviolet absorbance was detected at $290 \mathrm{~nm}$.

\section{Antioxidant properties of seaweed extracts}

The antioxidant activity of crude and fractions obtained from $S$. horneri were analyzed as a measurement of DPPH and alkyl radical scavenging activities as described by Nanjo et al. (1996) and Hiramoto et al. (1993). The measurements were taken by an electron spin resonance spectrometer (JES-FA200; Jeol, Tokyo, Japan) at $25^{\circ} \mathrm{C}$.

\section{Cell experiments}

Cell culture. RAW 264.7 cells were cultured in a DMEM medium supplemented with $10 \%$ heat-inactivated FBS and $1 \%$ streptomycin and penicillin $\left(100 \mu \mathrm{gL}^{-1}\right)$. The cells were maintained under humidified conditions at $37^{\circ} \mathrm{C}$ with $5 \% \mathrm{CO}_{2}$. RAW 264.7 cells were periodically subcultured and cells under exponential growth were used for experiments.

Evaluating the inhibition of NO production and cytotoxicity of seaweed extracts on lipopolysaccharide (LPS)-stimulated RAW 264.7 macrophages. RAW 264.7 cells $\left(1 \times 10^{5}\right.$ cells $\left.\mathrm{mL}^{-1}\right)$ were seeded in 24 well plates and incubated for $24 \mathrm{~h}$. Then the cells were treated with 25 $\mu \mathrm{L}$ seaweed extracts $\left(12.5,25\right.$, or $\left.50 \mu \mathrm{g} \mathrm{mL} \mathrm{m}^{-1}\right)$ and stimulated with LPS (final concentration $=1 \mu \mathrm{g} \mathrm{mL}^{-1}$ ). Following a 24-h incubation period, the NO levels of the culture media were determined by Griess assay as described by Leiro et al. (2002) and the cell viability was determined using the MTT assay as described by Mosmann (1983) with some minor modifications. Absorbance was measured by a microplate reader (Bio-Tek Instruments Inc., Winooski, VT, USA).

\section{Statistical analysis}

All the data were expressed as the mean \pm standard of 3 determinations. The results were analyzed by using the SPSS ver. 20 statistical analysis package (IBM Corp., Armonk, NY, USA). Duncan's multiple range test was used to determine mean separation. p-value $<0.05$ was considered as statistically significant.

\section{RESULTS}

\section{Proximate composition of seaweeds}

In the present study, we compared the proximate composition of two seaweed strains, S. horneri Korea (SK) and China (SC). According to the results, ash and moisture contents were ranged between $10.69-14.16 \%$ and 7.52-9.52\%, respectively (Table 1). The ash and moisture contents of SC were higher than SK. In parallel to these observations, Zhang et al. (2017) have reported that $S$. horneri collected along the coast of Heishijiao, Dalian contain around $8.8 \%$ ash as inorganic matters in the dried S. horneri biomass. 

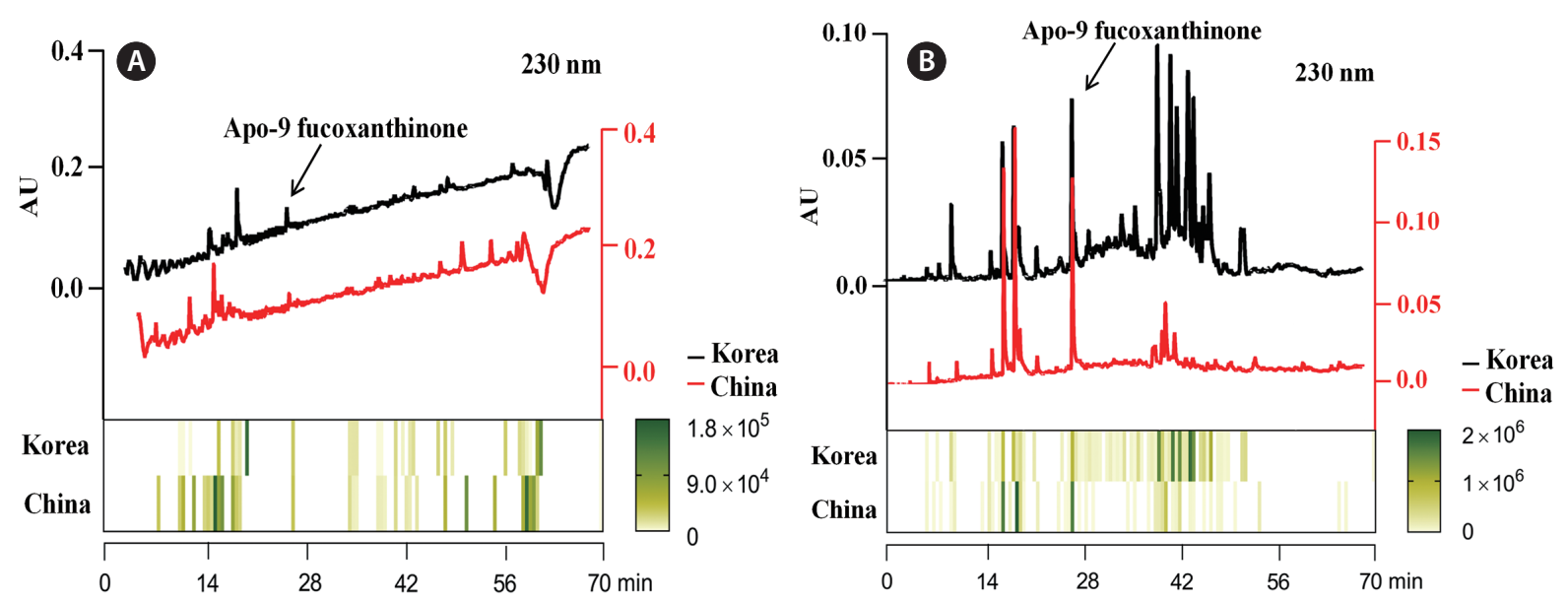

Fig. 1. High-performance liquid chromatography chromatogram of the $80 \%$ methanolic extracts (A) and the chloroform fraction separated from $80 \%$ methanolic extracts (B). Relevance heat maps of the absorbance for each sample obtained using GraphPad Prism 7.03 software.

\section{Preparation of $\mathbf{8 0} \%$ methanol extracts and frac- tionation}

Extraction of seaweeds with $80 \%$ methanol and further purification with solvent-solvent partition chromatography is considered as one of the best methods that can be applied for recovering bioactive phlorotannins from brown seaweeds (Ko et al. 2013). In order to study the bioactivities of two $S$. horneri strains first, seaweeds were extracted with $80 \%$ methanol and partially purified using solvent-solvent partition chromatography. From the $50 \mathrm{~g}$ of freeze dried SK and SC strains, we obtained $6.10 \mathrm{~g}$ and $3.30 \mathrm{~g}$ crude samples, respectively from $80 \%$ methanol extraction process. Even though SK had lower extraction efficiency its chloroform $(0.74 \%)$ and ethyl acetate fraction $(0.54 \%)$ was higher than the chloroform $(0.49 \%)$ and ethyl acetate fraction $(0.36 \%)$ obtained from SC. Af- ter extracting SK and SC into 80\% methanol solution, we compared the total phenol content, polysaccharides and sterol content in the methanolic extracts of SK (MSK) and SC (MSC). According to the results, polyphenol content of MSC $(7.09 \pm 0.17)$ was lower than MSK $(7.70 \pm 0.08)$ (Table 2). However, according to the polyphenol content both seaweed has slightly similar amounts of polyphenol compositions. These results indicate similarities with the results provided by Heo et al. (2005). It can, therefore, be concluded that polyphenol content of both strains does not indicate much deviation. Polysaccharide contents were higher in MSK compared to MSC and sterols were higher in MSC compared to MSK. Previous reports have indicated that $S$. horneri consisted of vitamins, amino acids, and polysaccharides and it has a potential to utilized as a food source (Sanjeewa et al. 2017a).

Table 1. Proximate chemical composition of Korean and China Sargassum horneri strains

\begin{tabular}{cccccc}
\hline Sample & Polysaccharide (\%) & Proteins (\%) & Lipids (\%) & Ash (\%) & Moisture (\%) \\
\hline Korean & $60.43 \pm 3.31$ & $19.29 \pm 1.30$ & $2.07 \pm 0.36$ & $10.69 \pm 1.34$ & $7.52 \pm 1.87$ \\
China & $58.24 \pm 2.72$ & $16.77 \pm 2.31$ & $1.31 \pm 0.32$ & $14.16 \pm 1.27$ & $9.52 \pm 2.01$ \\
\hline
\end{tabular}

Values are presented as mean \pm standard deviation $(n=3)$.

Table 2. General components of $80 \%$ methanolic extract of Sargassum horneri Korea and China strains

\begin{tabular}{|c|c|c|c|}
\hline Sample & Polyphenol (g $\left.100 \mathrm{~g}^{-1}\right)$ & Polysaccharides $\left(\mathrm{g} 100 \mathrm{~g}^{-1}\right)$ & Sterol $\left({\left.\mathrm{g} 100 \mathrm{~g}^{-1}\right)}\right.$ \\
\hline Korean & $7.70 \pm 0.08$ & $12.68 \pm 0.10$ & $7.50 \pm 0.53$ \\
\hline China & $7.09 \pm 0.17$ & $8.77 \pm 1.21$ & $11.14 \pm 0.10$ \\
\hline
\end{tabular}

Values are presented as mean \pm standard deviation $(n=3)$. 


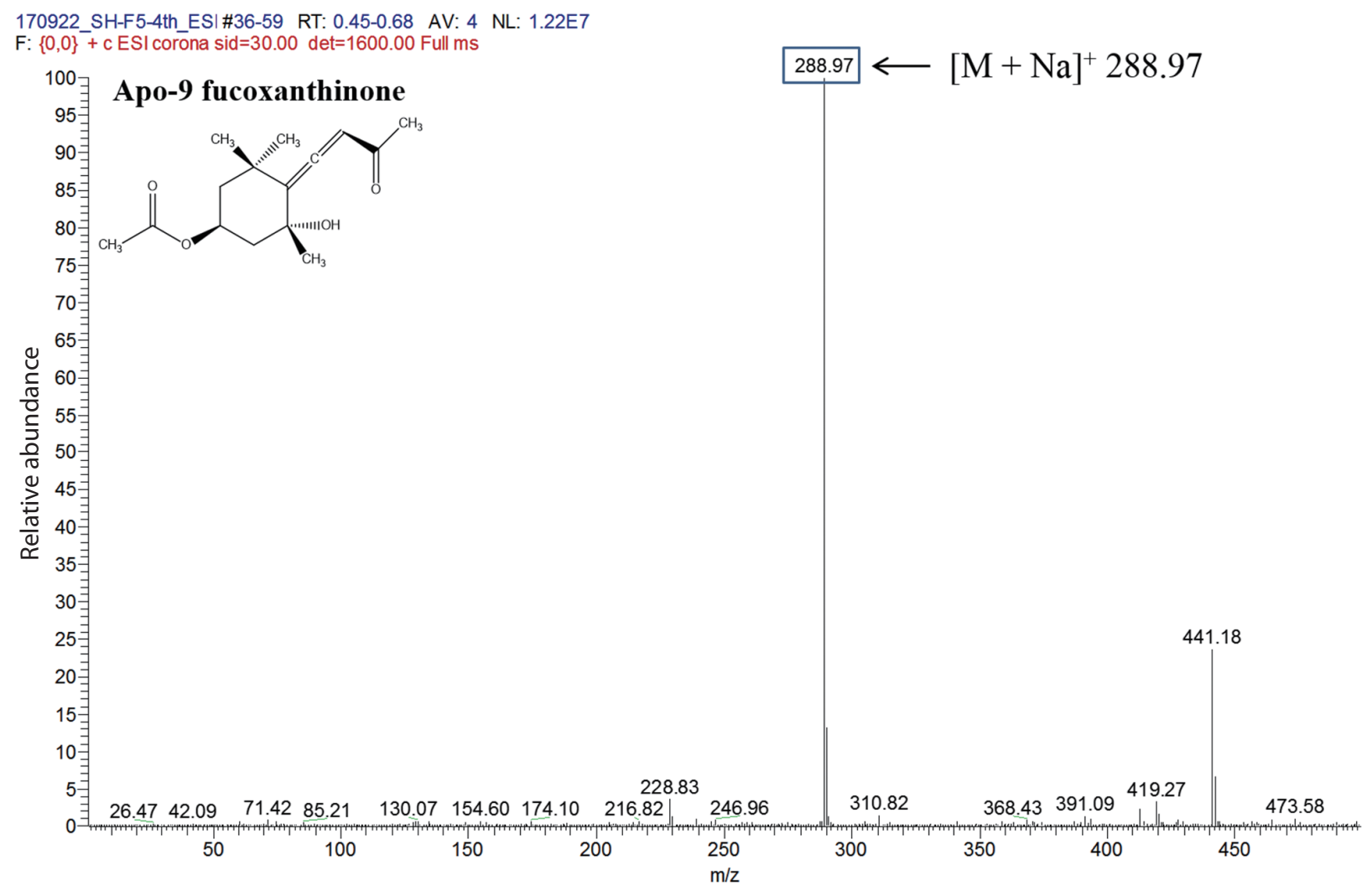

Fig. 2. Mass spectra of the Apo-9 fucoxanthinone isolated from Sargassum horneri.

\section{HPLC-analysis of crude and fractionated extracts of SK and SC}

The $80 \%$ MSK and MSC, as well as the chloroform fractions (CMSK and CMSC) obtained from the methanolic extracts, were compared using HPLC-spectrum. First, we compared the HPLC-chromatograms and heat maps of MSK and MSC (Fig. 1A). According to the results, both strains might contain similar biomolecules but in different quantities due to the difference observed in the height of the respective peaks. Specifically, we observed one major peak at around $27.5 \mathrm{~min}$ in the methanolic extracts and both are similar in the particular compound by the peak heights and heat map results. In the HPLCchromatograms and heat maps of the chloroform fractions (Fig. 1B), one major peak developed in the HPLCchromatograms was observed at $27.5 \mathrm{~min}$, but high in SC than in SK. The major peak was identified to Apo-9 fucoxanthinone by liquid chromatography-mass spectrometry (Fig. 2). In addition, some other major peaks on CMSC chromatogram around at 10-25 min, CMSK at around 36-47 min were found but not identified.

\section{Antioxidant properties of crude and fraction- ated extracts of SK and SC}

Electron spin resonance spectrometry (ESR) is one of the most common technique used to identify the radical scavenging effect of compounds. Spin trapping is the direct and validated method to detect highly reactive free radicals generated for the small time periods (Ahn et al. 2007). In this study, we compared the radical scavenging effects of crude samples and their fractions (Table 3). Alkyl radical scavenging activity of MSK and MSC, as well as their fractions, were higher than that of the DPPH radical scavenging effect. Interestingly, $\mathrm{IC}_{50}$ value of fractions separated from both samples were less than $50 \mu \mathrm{g} \mathrm{mL} \mathrm{m}^{-1}$. These observations suggest that the metabolites present in both seaweed strains have strong antioxidant compounds. Comparatively the radical scavenging effect of SK was slightly higher than SC. According to ESR analysis, we concluded that SK has better antioxidant capacity than SC. 


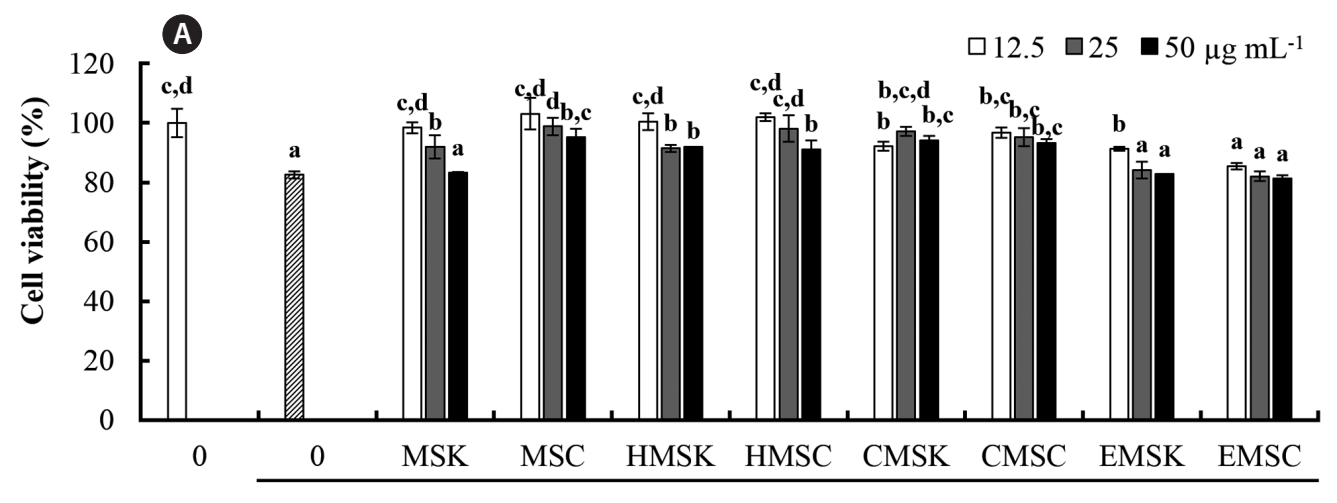

LPS $\left(1 \mu \mathrm{g} \mathrm{mL} \mathrm{m}^{-1}\right)$

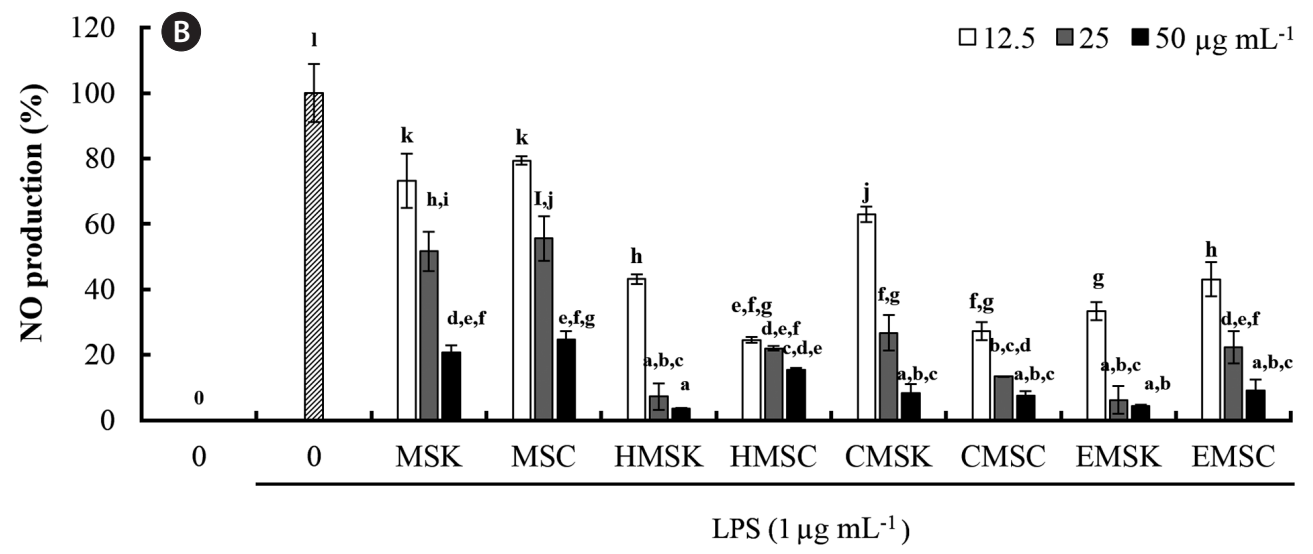

Fig. 3. Anti-inflammatory effects of $80 \%$ methanolic extract and its fractions separated from Sargassum horneri Korea (SK) and China (SC) strains. (A) The cytotoxicity of extracts on RAW 264.7 cells in the presence of lipopolysaccharide (LPS). The viability of cells without sample and LPS has been taken as reference (100\%). (B) The dose-dependent inhibition of seaweed extracts against nitric oxide (NO) production in LPS-stimulated RAW 264.7 macrophages. The level of NO production is expressed as percentages of that of the group exposed to the LPS. Data points and bars represent the arithmetic means \pm standard error $(n=3)$. Means not sharing a common letter indicates significant difference between the groups ( $\mathrm{p}<0.05$ by Duncan's multiple range test). MSK, $80 \%$ methanol extract of SK; MSC, $80 \%$ methanol extract of SC; HMSK, hexane fraction of MSK; HMSC, hexane fraction of MSC; CMSK, chloroform fraction of MSK; CMSC, chloroform fraction of MSC; EMSK, ethyl acetate fraction of MSK; EMSC, ethyl acetate fraction of MSC.

Table 3. Yield and antioxidant properties of $80 \%$ methanolic extract of Sargassum horneri Korea (SK) and China (SC) strains

\begin{tabular}{lccc}
\hline & Yield (dry weight, \%) & DPPH $\left(\mathrm{IC}_{50} \mathbf{~ m g ~ m L}^{-1}\right)$ & Alkyl $\left(\mathrm{IC}_{50} \mathrm{mg} \mathrm{mL}^{-1}\right)$ \\
\hline MSK & $7.10 \pm 0.50$ & $1.12 \pm 0.27$ & $0.17 \pm 0.03$ \\
MSC & $11.52 \pm 0.98$ & $1.23 \pm 0.18$ & $0.24 \pm 0.07$ \\
HMSK & $0.17 \pm 0.03$ & $<5$ & $<5$ \\
HMSC & $2.32 \pm 0.19$ & $<5$ & $0.04 \pm 0.01$ \\
CMSK & $0.74 \pm 0.01$ & $2.71 \pm 0.13$ & $0.04 \pm 0.01$ \\
CMSC & $0.49 \pm 0.02$ & $2.32 \pm 0.09$ & $0.02 \pm 0.01$ \\
EMSK & $0.54 \pm 0.01$ & $1.46 \pm 0.03$ & $0.01 \pm 0.00$ \\
EMSC & $0.36 \pm 0.04$ & $2.09 \pm 0.07$ & \\
\hline
\end{tabular}

Values are presented as mean \pm standard deviation $(n=3)$.

DPPH, 2,2-diphenyl-1-picrylhydrazy; MSK, methanol extract of SK; MSC, methanol extract of SC; HMSK, hexane fraction of MSK; HMSC, hexane fraction of MSC; CMSK, chloroform fraction of MSK; CMSC, chloroform fraction of MSC; EMSK, ethyl acetate fraction of MSK; EMSC, ethyl acetate fraction of MSC. 


\section{Anti-inflammatory effects of seaweed extracts and its fractions on LPS-stimulated RAW 264.7 cells}

LPS-stimulated RAW 264.7 cells were used to compare the anti-inflammatory properties of SK and SC. According to Fig. 3A concentrations of 12.5-50 $\mu \mathrm{g} \mathrm{mL}^{-1}$ did not indicate any significant cytotoxic effect on RAW 264.7 cells compared to the LPS alone treatment. Thus, authors compared NO inhibitory effects of two seaweed strains using the same concentrations of methanol extract and its fractions (Fig. 3B). According to the results, MSK and MSC inhibited the LPS-stimulated NO production from RAW 264.7 cells. Specifically, all fractions had increased NO suppressive effect on LPS-stimulated RAW 264.7 cells than their methanol extracts. In general, it is clear that both SK and SC has strong anti-inflammatory effects on LPS-stimulated RAW 264.7 cells.

\section{DISCUSSION}

Sargassum horneri is a common seaweed in China, which grows as solid substrates in the sub-littoral region of the Chinese coast. The large-scale drift biomass of $S$. horneri was moved to Jeju Island from Chinese coast (Su et al. 2018). Therefore, both SK and SC are present in large amounts along the shores of Jeju Island, South Korea (Sanjeewa et al. 2017a). Due to high amount of the seaweed it makes adverse effects on the shores of Jeju by effecting to its beauty, economic losses to the fisheries industries as well as creating imbalance in marine habitats. But among the marine organisms, S. horneri is one of the promising sources for isolating bioactive compounds. The secondary metabolites of Sargassum sp. have several structural classes such as steroids, chromanols, glycerides, and fatty acids, which are mostly obtained from chloroform fraction (Lee et al. 2013). In the same manner, Cho (2013) reported six different chromanols isolated from S. horneri have anti-fouling effect against Mytilus edulis (Cho 2013). In addition, it has been reported that chromanols, chlorophyll, fucoxanthin, as well as phlorotannins separated from S. horneri possess bioactive properties such as anti-inflammation, anti-allergic, and anti-oxidant properties (Heo et al. 2005, 2010, Yoshioka et al. 2014). Most of previous studies focus only on one $S$. horneri strain growing in a limited area. In this study, however we compared the general components and bio- activates of SK and SC collected along the coasts of Jeju Island.

According to the results, SK had lower extraction efficiency compared to SC. After the solvent-solvent partition chromatography, more than $95 \%$ of both strains moved into the hexane fraction ( 20\%) and water fraction $(\sim 75 \%)$. Even though MSK had a low extraction efficiency compared to MSC, the amount of chloroform and ethyl acetate fractions were higher in MSK. However, both strains had similar NO inhibitory effects on LPS-stimulated RAW 264.7 cells, and they indicated much similar radical scavenging properties. The HPLC chromatogram analysis confirmed that the methanolic extracts and their chloroform fractions of both strains contain Apo-9 fucoxanthinone as one of their major constituents with similar amount and chromatogram pattern. Thus, we expect that Apo-9 fucoxanthinone, a major peak in the chloroform fraction, is involved in the strong anti-inflammatory and radical scavenging effects. In fact, Apo-9 fucoxanthinoe has been shown to have strong anti-inflammatory activity and DNA damage protection effects against cigarette smoke (Jang et al. 2016, Kim et al. 2018). Many of the previous studies have reported that chloroform and ethyl acetate fractions are rich in bioactive compounds like phlorotannins and chromanols (Fenical and McConnell 1975, Heo et al. 2010, Wang et al. 2012). Therefore, we conclude that SC could be utilized as a raw material to isolate bioactive compounds similar to SK components.

In the present study, we demonstrated the $80 \%$ methanol extract of both strains seaweeds had similar bioactive properties. However, the extraction efficiency of SK and SC had considerable differences. Even SC had high extraction efficiency on $80 \%$ methanol, its chloroform and ethyl acetate fractions had smaller yields compared to those of SK. Thus, for the industrial level applications, it is better to select SK over SC. However, SC might be sufficient for industrial utilisation, due to its strong antiinflammatory and antioxidant activities as well as Apo-9 fucoxanthinone which is available in China strain.

\section{ACKNOWLEDGEMENTS}

This research was supported by Basic Science Research Program through the National Research Foundation of Korea (NRF) funded by the Ministry of Science, ICT \& Future Planning (NRF-2015R1D1A1A09060048). 


\section{REFERENCES}

Ahn, G. -N., Kim, K. -N., Cha, S. -H., Song, C. -B., Lee, J., Heo, M. -S., Yeo, I. -K., Lee, N. -H., Jee, Y. -H., Kim, J. -S., Heu, M. -S. \& Jeon, Y. -J. 2007. Antioxidant activities of phlorotannins purified from Ecklonia cava on free radical scavenging using ESR and $\mathrm{H}_{2} \mathrm{O}_{2}$-mediated DNA damage. Eur. Food Res. Technol. 226:71-79.

Association of Official Analytical Chemists. 1998. Official methods of analysis of AOAC international. 16th ed. Vol. 2. Association of Official Analytical Chemists, Washington, DC, $1018 \mathrm{pp}$.

Chandler, S. F. \& Dodds, J. H. 1983. The effect of phosphate, nitrogen and sucrose on the production of phenolics and solasodine in callus cultures of solanum laciniatum. Plant Cell Rep. 2:205-208.

Cho, J. Y. 2013. Antifouling chromanols isolated from brown alga Sargassum horneri. J. Appl. Phycol. 25:299-309.

Choi, W. -C., Reid, S. N. S., Ryu, J. -K., Kim, Y., Jo, Y. -H. \& Jeon, B. H. 2016. Effects of $\gamma$-aminobutyric acid-enriched fermented sea tangle (Laminaria japonica) on brain derived neurotrophic factor-related muscle growth and lipolysis in middle aged women. Algae 31:175-187.

DuBois, M., Gilles, K. A., Hamilton, J. K., Rebers, P. A. \& Smith, F. 1956. Colorimetric method for determination of sugars and related substances. Anal. Chem. 28:350-356.

Esser, N., Legrand-Poels, S., Piette, J., Scheen, A. J. \& Paquot, N. 2014. Inflammation as a link between obesity, metabolic syndrome and type 2 diabetes. Diabetes Res. Clin. Pract. 105:141-150.

Fenical, W. \& McConnell, O. 1975. Chromazonarol and isochromazonarol, new chromanols from the brown seaweed Dictyopteris undulata (zonarioides). Experientia 31:1004-1005.

Fernando, I. P. S., Kim, H. -S., Sanjeewa, K. K. A., Oh, J. -Y., Jeon, Y. -J. \& Lee, W. W. 2017a. Inhibition of inflammatory responses elicited by urban fine dust particles in keratinocytes and macrophages by diphlorethohydroxycarmalol isolated from a brown alga Ishige okamurae. Algae 32:261-273.

Fernando, I. P. S., Kim, M., Son, K. -T., Jeong, Y. \& Jeon, Y. -J. 2016. Antioxidant activity of marine algal polyphenolic compounds: a mechanistic approach. J. Med. Food 19:615-628

Fernando, I. P. S., Sanjeewa, K. K. A., Samarakoon, K. W., Lee, W. W., Kim, H. -S., Kim, E. -A., Gunasekara, U. K. D. S. S., Abeytunga, D. T. U., Nanayakkara, C., de Silva, E. D., Lee, H. -S. \& Jeon, Y. -J. 2017b. FTIR characterization and antioxidant activity of water soluble crude polysaccharides of Sri Lankan marine algae. Algae 32:75-86.
Heo, S. -J., Cha, S. -H., Lee, K. -W., Cho, S. K. \& Jeon, Y. -J. 2005. Antioxidant activities of chlorophyta and phaeophyta from Jeju Island. Algae 20:251-260.

Heo, S. -J., Yoon, W. -J., Kim, K. -N., Ahn, G. -N., Kang, S. -M., Kang, D. -H., Affan, A., Oh, C., Jung, W. -K. \& Jeon, Y. -J. 2010. Evaluation of anti-inflammatory effect of fucoxanthin isolated from brown algae in lipopolysaccharide-stimulated RAW 264.7 macrophages. Food Chem. Toxicol. 48:2045-2051.

Hiramoto, K., Johkoh, H., Sako, K. -I. \& Kikugawa, K. 1993. DNA breaking activity of the carbon-centered radical generated from 2,2'-azobis(2-amidinopropane) hydrochloride (AAPH). Free Radic. Res. Commun. 19:323-332.

Jang, J. -H., Lee, J. -H., Chand, H. S., Lee, J. -S., Lin, Y., Weathington, N., Mallampalli, R., Jeon, Y. -J. \& Nyunoya, T. 2016. Apo-9'-fucoxanthinone extracted from Undariopsis peteseniana protects oxidative stress-mediated apoptosis in cigarette smoke-exposed human airwat epithelial cells. Mar. Drugs 14:140.

Kim, E. -A., Kim, S. -Y., Ye, B. -R., Kim, J., Ko, S. -C., Lee, W. W., Kim, K. -N., Choi, I. -W., Jung, W. -K. \& Heo, S. -J. 2018. Anti-inflammatory effect of Apo-9'-fucoxanthinone via inhibition of MAPKs and NF-kB signaling pathway in LPS-stimulated RAW 264.7 macrophges and zebrafish model. Int. Immunopharmacol. 59:339-346.

Ko, S. -C., Lee, M., Lee, J. -H., Lee, S. -H., Lim, Y. \& Jeon, Y. -J. 2013. Dieckol, a phlorotannin isolated from a brown seaweed, Ecklonia cava, inhibits adipogenesis through AMP-activated protein kinase (AMPK) activation in 3T3L1 preadipocytes. Environ. Toxicol. Pharmacol. 36:12531260.

Kubo, N., Douke, A., Nishigaki, T. \& Tsuji, G. 2016. Development and characterization of simple sequence repeat markers for genetic analyses of Sargassum horneri (Sargassaceae, Phaeophyta) populations in Kyoto, Japan. J. Appl. Phycol. 29:1729-1733.

Kundu, S. \& Kawamura, K. 2014. Seasonal variations of stable carbon isotopic composition of bulk aerosol carbon from Gosan site, Jeju Island in the East China Sea. Atmos. Environ. 94:316-322.

Lee, J. -H., Ko, J. -Y., Samarakoon, K., Oh, J. -Y., Heo, S. -J., Kim, C. -Y., Nah, J. -W., Jang, M. -K., Lee, J. -S. \& Jeon, Y. -J. 2013. Preparative isolation of sargachromanol E from Sargassum siliquastrum by centrifugal partition chromatography and its anti-inflammatory activity. Food Chem. Toxicol. 62:54-60.

Leiro, J., Álvarez, E., García, D. \& Orallo, F. 2002. Resveratrol modulates rat macrophage functions. Int. Immunopharmacol. 2:767-774.

Lin, M. T. \& Beal, M. F. 2006. Mitochondrial dysfunction and 
oxidative stress in neurodegenerative diseases. Nature 443:787-795.

Mosmann, T. 1983. Rapid colorimetric assay for cellular growth and survival: application to proliferation and cytotoxicity assays. J. Immunol. Methods 65:55-63.

Nanjo, F., Goto, K., Seto, R., Suzuki, M., Sakai, M. \& Hara, Y. 1996. Scavenging effects of tea catechins and their derivatives on 1,1-diphenyl-2-picrylhydrazyl radical. Free Radic. Biol. Med. 21:895-902.

Pang, S. J., Liu, F., Shan, T. F., Gao, S. Q. \& Zhang, Z. H. 2009. Cultivation of the brown alga Sargassum horneri: sexual reproduction and seedling production in tank culture under reduced solar irradiance in ambient temperature. J. Appl. Phycol. 21:413-422.

Park, H. -J., Lee, M. -S., Shim, H. S., Lee, G. -R., Chung, S. Y., Kang, Y. M., Lee, B. -J., Seo, Y. B., Kim, K. S. \& Shim, I. 2016. Fermented Saccharina japonica (Phaeophyta) improves neuritogenic activity and TMT-induced cognitive deficits in rats. Algae 31:73-84.

Pesic, M. \& Greten, F. R. 2016. Inflammation and cancer: tissue regeneration gone awry. Curr. Opin. Cell Biol. 43:5561.

Preeprame, S., Hayashi, K., Lee, J. -B., Sankawa, U. \& Hayashi, T. 2001. A novel antivirally active fucan sulfate derived from an edible brown alga, Sargassum horneri. Chem. Pharm. Bull. (Tokyo) 49:484-485.

Sanjeewa, K. K. A., Fernando, I. P. S., Kim, E. -A., Ahn, G., Jee, Y. \& Jeon, Y. -J. 2017a. Anti-inflammatory activity of a sulfated polysaccharide isolated from an enzymatic digest of brown seaweed Sargassum horneri in RAW 264.7 cells. Nutr. Res. Pract. 11:3-10.

Sanjeewa, K. K. A., Fernando, I. P. S., Kim, S. -Y., Kim, H. -S., Ahn, G., Jee, Y. \& Jeon, Y. -J. 2018. In vitro and in vivo anti-inflammatory activities of high molecular weight sulfated polysaccharide: containing fucose separated from Sargassum horneri: short communication. Int. J.
Biol. Macromol. 107:803-807.

Sanjeewa, K. K. A., Fernando, I. P. S., Samarakoon, K. W., Lakmal, H. H. C., Kim, E. -A., Kwon, O. -N., Dilshara, M. G., Lee, J. -B. \& Jeon, Y. -J. 2016. Anti-inflammatory and anti-cancer activities of sterol rich fraction of cultured marine microalga Nannochloropsis oculata. Algae 31: 277-287.

Sanjeewa, K. K. A., Lee, J. -S., Kim, W. -S. \& Jeon, Y. -J. $2017 b$. The potential of brown-algae polysaccharides for the development of anticancer agents: an update on anticancer effects reported for fucoidan and laminaran. Carbohydr. Polym. 177:451-459.

Su, L., Shan, T., Pang, S. \& Li, J. 2018. Analyses of the genetic structure of Sargassum horneri in the Yellow Sea: implications of the temporal and spatial relations among floating and benethic populations. J. Appl. Phycol. 30:1417-1424.

Wang, G., Sun, J., Liu, G., Wang, L., Yu, J., Liu, T., Chi, S., Liu, C., Guo, H. \& Wang, X. 2014. Comparative analysis on transcriptome sequencings of six Sargassum species in China. Acta Oceanol. Sin. 33:37-44.

Wang, T., Jónsdóttir, R., Liu, H., Gu, L., Kristinsson, H. G., Raghavan, S. \& Ólafsdóttir, G. 2012. Antioxidant capacities of phlorotannins extracted from the brown algae Fucus vesiculosus. J. Agric. Food Chem. 60:5874-5883.

Wijesinghe, W. A. J. P. \& Jeon, Y. -J. 2012. Enzyme-assistant extraction (EAE) of bioactive components: a useful approach for recovery of industrially important metabolites from seaweeds: a review. Fitoterapia 83:6-12.

Yoshioka, H., Ishida, M., Nishi, K., Oda, H., Toyohara, H. \& Sugahara, T. 2014. Studies on anti-allergic activity of Sargassum horneri extract. J. Funct. Foods 10:154-160.

Zhang, L., Zhao, C., Shi, D., Hu, W., Wei, J. \& Chang, Y. 2017. Gulfweed Sargassum horneri is an alternative diet for aquaculture of juvenile sea urchins Strongylocentrotus intermedius in summer. Aquac. Int. 25:905-914. 